\title{
Feynman, dissipação e computação quântica
}

\author{
Feynman, dissipation and quantum computation
}

\author{
Amir Caldeira*10
}

\author{
${ }^{1}$ Universidade Estadual de Campinas, Instituto de Física Gleb Wataghin, Campinas, SP, Brasil
}

Recebido em 13 de Dezembro, 2017. Aceito em 15 de Fevereiro, 2018.

\begin{abstract}
Neste artigo vamos procurar abordar a influência, direta ou indireta, que R. P. Feynman teve em duas áreas extremamente importantes para o desenvolvimento da física em nossos dias; a dissipação e a computação quântica. Em particular, vamos ainda descrever como estas duas linhas de pesquisa se conectam e nos ensinam como lidar com as limitações de um dos grandes desafios tecnológicos do momento; a construção de um computador quântico universal. Mais uma vez, Feynman aparece como figura central na origem da formulação de métodos e ideias que norteiam inúmeros pesquisadores que atuam nestas áreas do conhecimento.
\end{abstract}

Palavras-chave: dissipação quântica; computação quântica; Feynman; computador quântico.

In this article we address the influence that R. P. Feynman had in two áreas extremely important for the development of physics nowadays: the dissipation and the quantum computation. In particular, we also describe how these two lines of research connect and teach us how to deal with the limitations of one of the major technological challenges of the moment: the construction of a universal quantum computer. Once again, Feynman appears as the central figure at the origin of the formulation of methods and ideas that guide many researchers working in these areas of knowledge.

Keywords: quantum dissipation; quantum computation; Feynman; quantum computer.

\section{Introdução}

Ao receber o convite para colaborar com este volume comemorativo do centésimo aniversário de R. P. Feynman da Revista Brasileira de Ensino de Física, senti-me obviamente lisonjeado por ter sido escolhido para escrever sobre parte do trabalho de um dos maiores cientistas do século XX. Entretanto, logo que comecei a planejar o que exatamente escrever e como abordar o tema que me foi sugerido - o papel de Feynman na dissipação e na computação quântica - dei conta que a tarefa não seria trivial. Não pelo volume do material produzido por Feynman nestas linhas de pesquisa que, diga-se de passagem, não é grande, mas pela profundidade com que ele tratava tudo aquilo em que se envolvia.

Feynman seguramente não figurava entre os físicos que detêm o controle de determinada técnica e buscam problemas onde elas possam ser convenientemente aplicadas. Ao contrário desta postura, a que ele adotava era buscar problemas fundamentais para os quais pudesse dar a sua contribuição, independentemente da abordagem a ser utilizada, no que, sem dúvida, teve pleno sucesso ao longo de toda a sua carreira.

Sua meta era sempre entender a física envolvida no fenômeno em questão. A propósito, até mesmo problemas cuja solução já era conhecida Feynman procurava

*Endereço de correspondência: caldeira@ifi.unicamp.br resolver de sua maneira e, como resultado, muitas vezes gerou métodos alternativos de solução que tiveram desdobramentos de enorme importância no desenvolvimento posterior de várias áreas da física. Um exemplo bem conhecido disso é a sua formulação de integrais de trajetória (ou integrais funcionais) [1] da mecânica quântica.

Nesta formulação, o propagador do estado físico que descreve um sistema quântico evoluindo entre dois pontos do espaço-tempo pode ser descrito como uma "soma" de exponenciais complexas das ações clássicas ao longo de todos os caminhos geométricos que unem estes dois pontos. Esta formulação, como veremos adiante, ainda que complicada e desnecessária para vários problemas elementares de mecânica quântica, torna-se extremamente útil para tratar sistemas quânticos com um número infinito de graus de liberdade, como é o caso de uma teoria quântica de campos, ou em várias outras aplicações [1].

O caso da computação quântica não é diferente. O interesse de Feynman pela teoria da computação começa ainda durante o projeto Manhattan [2] e culmina, já no começo da década de 1980, com seu envolvimento na preparação de um curso onde aborda, entre outros tópicos convencionais desta area, questões relativas aos limites físicos da computação, o que naturalmente já o conduzia aos possíveis efeitos da mecânica quântica na teoria da computação.

Os dois tópicos que vou abordar neste artigo foram desenvolvidos independentemente, mas, tão logo questio- 
namentos mais práticos vieram à tona, a convergência dos dois temas naturalmente ocorreu. Questões envolvendo a preservação da coerência quântica para a confecção de um processador quântico se tornaram cruciais e todo o estudo que vinha sendo desenvolvido em sistemas quânticos meso ou nanoscópicos (dissipativos por natureza) passou a integrar o conjunto dos temas fundamentais para as teorias recém-criadas da informação e computação quântica.

Como mencionei acima, a escolha da forma de apresentação do desenvolvimento destas áreas não é única e nem mesmo simples. Mas, para tentar exemplificar tudo o que foi feito durante os últimos 40 anos com relação a estes temas, optei por apresentar os passos que contribuiram para o progresso destas áreas em ordem cronológica. Desta forma acredito que o leitor terá uma visão mais ampla e abrangente destas questões. Em particular, pretendo evidenciar, quando pertinente, o papel desempenhado por Feynman neste processo

\section{Dissipação quântica}

Pode-se definir dissipação quântica como a perda de energia que um sistema quântico sofre de forma sistemática e irreversível quando não está isolado do seu meio ambiente. Entretanto, o que convenciona-se ser o paradigma da chamada dissipação quântica no jargão da comunidade científica no momento é o sistema quântico cuja dinâmica pode, no limite clássico (alta temperatura $T$ ), ser descrita pela equação de movimento:

$$
M \ddot{q}+\eta \dot{q}+V^{\prime}(q)=f(t),
$$

que é a consagrada equação de Langevin do movimento browniano.

$\mathrm{Na}$ equação acima $f(t)$ é uma força flutuante tal que $\langle f(t)\rangle=0$ e $\left\langle f(t) f\left(t^{\prime}\right)\right\rangle=2 \eta k_{B} T \delta\left(t-t^{\prime}\right)$, onde $M$ é a massa da partícula, $\eta$ é a constante de dissipação, $V(q)$ é um potencial externo, e $k_{B}$ a constante de Boltzmann.

Esta equação descreve o movimento de uma partícula em um fluido viscoso quando a massa $M>>m$, onde $m$ é a massa das moléculas do fluido, e estamos interessados em tempos bem mais longos que o tempo médio entre colisões moleculares.

Apesar de termos escolhido um modelo mecânico para exemplificar o movimento browniano, cabe enfatizar que há na natureza inúmeros sistemas cuja dinâmica pode ser descrita por equações do tipo (1). Um caso particularmente importante é o da dinâmica do fluxo magnético no interior de um diminuto anel supercondutor fechado por uma junção Josephson [3,4], comumente chamado de SQUID (supercondcting quantum interference device). Se a junção tem capacitância $C$ e resistência $R$ no seu estado normal, a equação obedecida pelo fluxo eletromagnético no seu interior é dada por

$$
C \ddot{\phi}+\frac{\dot{\phi}}{R}+U^{\prime}(\phi)=I_{f}(t)
$$

onde a corrente flutuante $I_{f}(t)$ é tal que $\left\langle I_{f}(t)\right\rangle=0$ e $\left\langle I_{f}(t) I_{f}\left(t^{\prime}\right)\right\rangle=2 k_{B} T R^{-1} \delta\left(t-t^{\prime}\right)$, o que representa o chamado "ruído branco". Aqui, $U(\phi)$ representa a energia eletromagnética armazenada no circuito e faz o papel da energia potencial em (1).

$\mathrm{Na}$ realidade foi este o sistema que originou todo o interesse na quantização de equações dissipativas. Os SQUIDs se mostravam candidatos ideais para o estudo do tunelamento de uma "partícula browniana" [5], mas não se conhecia nenhum método para quantizar um sistema dissipativo.

A razão para tal é que estes sistemas não são hamiltonianos e, portanto, as conhecidas relações canônicas de quantização não podem ser usadas diretamente. Este fato gerou ao longo de alguns anos muitos estudos sobre como estas relações deveriam ser modificadas ou generalizadas no sentido de se poder quantizar a equação de Langevin. Entretanto, todos os métodos sugeridos sempre acabavam apresentando problemas com alguns conceitos fundamentais da mecânica quântica como, por exemplo, o princípio da incerteza.

A solução deste problema consiste em se adotar uma abordagem mais pragmática onde se leva em conta que não há na natureza nenhum sistema dissipativo que não esteja acoplado com o seu meio ambiente. Desta forma pode-se considerar o meio como um segundo sistema (reservatório) ao qual o nosso sistema de interesse, a partícula browniana, está acoplado. Este novo sistema composto (o universo) é então um sistema hamiltoniano ao qual se pode aplicar as relações canônicas de quantização.

Neste ponto surge outro problema. Em geral não se conhece exatamente as interações do sistema de interesse com o seu meio ambiente e, mesmo se fossem conhecidas, um tratamento de primeiros princípios seria extremamente complicado devido à complexidade destes sistemas. Outra opção é adotar um modelo simples para o reservatório e seu acoplamento ao sistema de interesse de forma que a dinâmica clássica deste último resultante do seu acoplamento ao reservatório possa ser descrita por uma equação de Langevin [6, 7].

O modelo adotado é o de um conjunto de osciladores harmônicos não interagentes, mas acoplados ao sistema de interesse através de um acoplamento bilinear coordenada-coordenada como descrito na lagrangiana

$$
L=L_{S}+L_{I}+L_{R}+L_{C T},
$$

onde

$$
\begin{gathered}
L_{S}=\frac{1}{2} M \dot{q}^{2}-V(q), \\
L_{I}=\sum_{k} C_{k} q_{k} q, \\
L_{R}=\sum_{k} \frac{1}{2} m_{k} \dot{q}_{k}^{2}-\sum_{k} \frac{1}{2} m_{k} \omega_{k}^{2} q_{k}^{2}, \\
L_{C T}=-\sum_{k} \frac{1}{2} \frac{C_{k}^{2}}{m_{k} \omega_{k}^{2}} q^{2},
\end{gathered}
$$


que representam, respectivamente, o sistema de interesse, a interação, o reservatório e um contra-termo que adicionamos para preservar o potencial original $V(q)$ na equação de movimento resultante para $q(t)$ (1).

Visando a obtenção de (1) deve-se ainda definir a chamada função espectral $J(\omega)$ como

$$
J(\omega)=\frac{\pi}{2} \sum_{k} \frac{C_{k}^{2}}{m_{k} \omega_{k}} \delta\left(\omega-\omega_{k}\right),
$$

que nos permite transformar $\sum_{k} \longrightarrow \int d \omega$. Detalhes deste desenvolvimento podem ser encontrados, por exemplo, na referência [6].

A ideia do acoplamento ao banho de osciladores tem sido usada em diferentes contextos ao longo dos anos e uma de suas aplicações mais conhecidas é na descrição da relaxação de modos eletromagnéticos em cavidades [8], fundamental no desenvolvimento da teoria do laser. Adotando a chamada aproximação de onda girante (RWA, em inglês) este fenômeno é descrito convenientemente ao se usar a representação de estados coerentes para o campo eletromagnético. Entretanto, o nosso interesse aqui é na obtenção de equações de Langevin bem gerais o que nos leva a elaborar uma formulação quântica mais abrangente, apesar de seguirmos o mesmo procedimento usado em ótica quântica.

\subsection{Abordagem geral}

O que fizemos acima foi adotar um método que naturalmente nos leva a analisar a equação de movimento resultante do acoplamento do sistema ao banho de osciladores. Por outro lado, é sabido que há inúmeros efeitos em mecânica quântica, tunelamento por exemplo, para os quais a descrição mais apropriada seria através do conhecimento da evolução temporal do estado físico do sistema em questão. No caso de um sistema não-isolado o estado físico do sistema é dado pelo seu operador densidade reduzido que definimos em seguida.

A fim de estudar a dinâmica do nosso subsistema, vamos considerar a hamiltoniana correspondente à lagrangiana descrita acima (3),

$$
\mathcal{H}=\mathcal{H}_{0}(q, p)+\mathcal{H}_{I}\left(q, q_{k}\right)+\mathcal{H}_{R}\left(q_{k}, p_{k}\right)+\mathcal{H}_{C T}(q)
$$

Esta hamiltoniana nos permite escrever a evolução temporal do estado físico de todo o universo (sistema + banho) como

$$
|\Psi(t)\rangle=e^{-i \mathcal{H} t / \hbar}|\Psi(0)\rangle
$$

ou, se o estado físico inicial é conhecido por uma distribuição estatística $p_{\Psi}$, podemos generalizar esta evolução para o operador densidade total do sistema,

$$
\hat{\rho}(t) \equiv \sum_{\Psi} p_{\Psi}|\Psi(t)\rangle\langle\Psi(t)|=e^{-i \mathcal{H} t / \hbar} \hat{\rho}(0) e^{i \mathcal{H} t / \hbar},
$$

que pode ser usado para se calcular valores médios de operadores

$$
\langle\hat{O}(t)\rangle=\operatorname{tr}\{\hat{\rho}(0) \hat{O}(t)\}=\operatorname{tr}\{\hat{\rho}(t) \hat{O}(0)\} .
$$

Aqui, o traço é tomado no espaço de Hilbert formado pelo produto direto do espaços do sistema de interesse e do reservatório.

Como em geral estamos interessados em operadores (observáveis) que se referem apenas ao sistema $S, \hat{O}=$ $\hat{O}(q, p)$, temos

$$
\begin{aligned}
\langle\hat{O}(q, p)\rangle & =\operatorname{tr}_{R S}\{\hat{\rho}(t) \hat{O}\}=\operatorname{tr}_{S}\left\{\left[\operatorname{tr}_{R} \hat{\rho}(t)\right] \hat{O}\right\} \\
& =\operatorname{tr}_{S}\{\tilde{\rho}(t) \hat{O}\}
\end{aligned}
$$

onde $\operatorname{tr}_{S}$ e $\operatorname{tr}_{R}$ representam os traços parciais com respeito a $S$ e $R$, e

$$
\tilde{\rho}(t) \equiv \operatorname{tr}_{R} \hat{\rho}(t)
$$

é o operador densidade reduzido do sistema de interesse.

\subsection{Evolução em tempo real}

Escrevendo a expressão 14 na representação de coordenadas ( $(x, y)$ para a partícula e $(\mathbf{R}, \mathbf{Q})$ para o banho) e assumindo que o operador densidade inicial do universo possa ser escrito de forma separável como

$$
\hat{\rho}\left(x^{\prime}, \mathbf{R}^{\prime}, y^{\prime}, \mathbf{Q}^{\prime}, 0\right)=\hat{\rho}_{S}\left(x^{\prime}, y^{\prime}, 0\right) \hat{\rho}_{R}\left(\mathbf{R}^{\prime}, \mathbf{Q}^{\prime}, 0\right),
$$

pode-se mostrar [7] que

$$
\tilde{\rho}(x, y, t)=\iint d x^{\prime} d y^{\prime} \mathcal{J}\left(x, y, t ; x^{\prime}, y^{\prime}, 0\right) \tilde{\rho}\left(x^{\prime}, y^{\prime}, 0\right),
$$

onde

$$
\begin{aligned}
& \mathcal{J}\left(x, y, t ; x^{\prime}, y^{\prime}, 0\right) \\
& =\iiint d \mathbf{R}^{\prime} d \mathbf{Q}^{\prime} d \mathbf{R}\left\{K\left(x, \mathbf{R}, t ; x^{\prime}, \mathbf{R}^{\prime}, 0\right)\right. \\
& \left.\times K^{*}\left(y, \mathbf{R}, t ; y^{\prime}, \mathbf{Q}^{\prime}, 0\right) \tilde{\rho}_{R}\left(\mathbf{R}^{\prime}, \mathbf{Q}^{\prime}, 0\right)\right\}
\end{aligned}
$$

é conhecido como o superpropagador do operador densidade reduzido do sistema e

$$
K\left(x, \mathbf{R}, t ; x^{\prime}, \mathbf{R}^{\prime}, 0\right)=\left\langle x, \mathbf{R}\left|e^{-i \mathcal{H} t / \hbar}\right| x^{\prime}, \mathbf{R}^{\prime}\right\rangle,
$$

é o propagador do estado físico do universo. Em nosso caso consideraremos $\tilde{\rho}_{R}\left(\mathbf{R}^{\prime}, \mathbf{Q}^{\prime}, 0\right)$ como o estado de equilíbrio térmico do banho.

É exatamente neste ponto onde a contribuição de Feynman torna-se importante porque todos os propagadores envolvidos na expressão (17) podem ser representados por integrais funcionais 11 como

$$
\begin{aligned}
& K\left(x, \mathbf{R}, t ; x^{\prime}, \mathbf{R}^{\prime}, 0\right) \\
& =\int_{x^{\prime}}^{x} \int_{\mathbf{R}^{\prime}}^{\mathbf{R}} \mathcal{D} x\left(t^{\prime}\right) \mathcal{D} \mathbf{R}\left(t^{\prime}\right) \exp \left\{\frac{i}{\hbar} S\left[x\left(t^{\prime}\right), \mathbf{R}\left(t^{\prime}\right)\right]\right\},
\end{aligned}
$$


onde

$$
S\left[x\left(t^{\prime}\right), \mathbf{R}\left(t^{\prime}\right)\right]=\int_{0}^{t} L\left(x\left(t^{\prime}\right), \mathbf{R}\left(t^{\prime}\right), \dot{x}\left(t^{\prime}\right), \dot{\mathbf{R}}\left(t^{\prime}\right)\right) d t^{\prime}
$$

é a ação do sistema composto $R+S$. Assim, se usarmos (18) em 17) temos

$$
\begin{aligned}
& \mathcal{J}\left(x, y, t ; x^{\prime}, y^{\prime}, 0\right)=\int_{x^{\prime}}^{x} \int_{y^{\prime}}^{y} \mathcal{D} x\left(t^{\prime}\right) \mathcal{D} y\left(t^{\prime}\right) \\
& \times \exp \left\{\frac{i}{\hbar} \tilde{S}_{0}\left[x\left(t^{\prime}\right)\right]\right\} \exp \left\{-\frac{i}{\hbar} \tilde{S}_{0}\left[y\left(t^{\prime}\right)\right]\right\} \mathcal{F}\left[x\left(t^{\prime}\right), y\left(t^{\prime}\right)\right]
\end{aligned}
$$

onde $\tilde{S_{0}}\left[q\left(t^{\prime}\right)\right]$ representa a ação de sistema $S$ como funcional do caminho $q(t)$ somada à ação do contra-termo em (7) e $\mathcal{F}\left[x\left(t^{\prime}\right), y\left(t^{\prime}\right)\right]$ é o chamado funcional de influência. Este último é a média térmica no estado inicial do reservatório do produto de seu propagador quando atuado por uma força externa que surge da presença do sistema $S$ pelo seu complexo conjugado. Desta forma vemos que a evolução temporal do sistema acoplado a um banho térmico fica totalmente determinada pela auto-interação do sistema com ele mesmo via o acoplamento com o banho.

Esta forma de abordar o problema foi desenvolvida originalmente no trabalho de Feynman e Vernon [1,7, 9 e apresenta uma grande vantagem sobre as abordagens anteriormente propostas.

Tanto em ótica quântica quanto em várias outras aplicações, a ideia de se estudar a dinâmica do subsistema $S$ tomando o traço parcial nas variáveis do banho era também implementada, mas, devido às características específicas dos sistemas em questão, usava-se uma equação diferencial para o estudo da evolução temporal do subsistema, a equação de Fokker-Planck 10,11]. Entertanto, este procedimento só é válido se o sistema apresentar uma relaxação muito lenta se comparada às escalas de tempo do sistema. Em termos mais técnicos, esta é uma aproximação válida quando tratamos o acoplamento sistema-banho através de teoria de perturbações.

De posse da nova formulação e tendo em vista que em nossa modelagem, assim como na proposta por Feynman e Vernon, o reservatório e seu acoplamento ao sistema de interesse podem ser descritos por lagrangianas quadráticas, o funcional de influência adquire uma forma puramente quadrática em termos dos caminhos $x(t)$ e $y(t)$ oriundos da nossa representação de coordenadas do operador densidade reduzido de $S$. Desta forma, a dificuldade em resolver exatamente o problema reside na forma do potencial externo, $V(q)$, ao qual o sistema está sujeito ou na escolha feita para o estado inicial do sistema $\tilde{\rho}\left(x^{\prime}, y^{\prime}, 0\right)$ em 16$)$. No caso da partícula livre, do potencial linear ou do oscilador harmônico, o problema torna-se exatamente solúvel para pacotes iniciais gaussianos sem que se precise apelar para métodos perturbativos.
Usando o modelo proposto em (3) pudemos estudar o comportamento quântico de sistemas que obedecem, no limite clássico, equações do tipo (1) tanto para a partícula livre quanto para o oscilador harmônico a qualquer temperatura. No segundo caso ainda é possível se estudar tanto o caso subamortecido como o superamortecido, o que não seria possível na aproximação perturbativa que faz uso de $\gamma \equiv \eta / 2 m \ll \omega_{0}$, onde $\omega_{0}$ é a frequência natural do oscilador quando isolado.

Outro problema de grande interesse que é uma aplicação imediata da abordagem desenvolvida por Feynman e Vernon é o do estudo da destruição da coerência quântica entre dois pacotes gaussianos 7, 12]. Neste caso,

$$
\begin{aligned}
& \psi(x)=\psi_{1}(x)+\psi_{2}(x) \\
& =\tilde{\mathcal{N}}\left[\exp -\frac{x^{2}}{4 \sigma^{2}}+\exp -\frac{\left(x+x_{0}\right)^{2}}{4 \sigma^{2}}\right],
\end{aligned}
$$

o que implica em

$$
\begin{aligned}
& \rho\left(x^{\prime}, y^{\prime}, 0\right) \\
& =\rho_{1}\left(x^{\prime}, y^{\prime}, 0\right)+\rho_{2}\left(x^{\prime}, y^{\prime}, 0\right)+\rho_{\text {int }}\left(x^{\prime}, y^{\prime}, 0\right),
\end{aligned}
$$

onde $\rho_{\text {int }}\left(x^{\prime}, y^{\prime}, 0\right)=\psi_{2}\left(x^{\prime}\right) \psi_{1}^{*}\left(y^{\prime}\right)+\psi_{1}\left(x^{\prime}\right) \psi_{2}^{*}\left(y^{\prime}\right)$ representa a interferência entre os dois pacotes no instante inicial.

No caso de um oscilador fracamente amortecido, à medida que o tempo evolui, o pacote centrado em $x=$ 0 permanece praticamente inalterado enquanto que o inicialmente centrado em $x=-x_{0}$ executa movimento harmônico amortecido e, portanto, relaxa com uma taxa $\gamma=\eta / 2 m$. Entretanto, o termo de interferência apresenta uma relaxação que depende da preparação do estado inicial. No caso que estamos tratando, a taxa de relaxação deste termo é dada, a $T=0$, por $\Gamma=N \gamma$ onde $N=$ $x_{0}^{2} / 2 \sigma^{2}$. Ou seja, quanto maior a distância inicial entre os centros dos dois pacotes, mais rápido o termo de interferência irá desaparecer. Este é o conhecido fenômeno de decoerência (ou descoerência).

Apesar de Feynman e Vernon não terem usado o modelo (3) em toda a sua plenitude para, por exemplo, seguir a evolução temporal de um pacote gaussiano de um oscilador browniano quântico ou estudar a perda de coerência entre dois pacotes, eles produziram uma análise bem geral da teoria de meios dissipativos lineares e reobtiveram o famoso teorema de flutuação e dissipação 13 nestes sistemas.

\subsection{Tunelamento dissipativo: metaestabilidade}

A análise da evolução temporal do operador densidade reduzido do sistema via integração funcional foi sem dúvida um avanço na teoria do movimento browniano quântico, mas ainda não havia uma forma de se resolver o problema do tunelamento quântico de uma partícula browniana através de potenciais que apresentassem barreiras de energia.

Este problema era o que, de fato, havia despertado o nosso interesse no estudo do movimento browniano 
quântico e que podia ser testado experimentalmente através do tunelamento de fluxóides no interior dos SQUIDs descritos por (2). Nesta situação o "potencial" $U(\phi)$ apresentava várias barreiras e o fluxo eletromagnético podia tunelar para fora de uma configuração metaestável [5, 7]. Visando o estudo apenas do decaimento do estado metaestável aproximamos este potencial por $U(\phi) \approx a \phi^{2}-b \phi^{3}$ onde $a, b>0$.

Por outro lado, na segunda metade dos anos 1970, alguns artigos extremamente importantes foram publicados sobre o decaimento do vácuo falso em teoria quântica de campos 14,15. Este problema tratava basicamente de uma teoria de um campo escalar sujeito a um potencial com dois mínimos de energia ligeiramente distintos. Se o sistema estivesse inicialmente no mínimo de energia mais alta ele poderia decair via tunelamento quântico para a configuração de energia mais baixa através da formação de bolhas desta nova configuração. Dependendo do tamanho que a bolha fosse formada, ela sumiria ou expandiria levando todo o sistema para a configuração energeticamente mais favorável. Ou seja, o sistema poderia decair por um mecanismo de nucleação quântica 7 .

Aqui, novamente a formulação de Feynman da mecânica quântica entrava em cena. Devido ao fato de o sistema apresentar um número infinito de graus de liberdade, o uso direto de "funcionais de onda" se mostrava extremamente complicado. Assim, os autores aplicaram a ideia da representação de integrais funcionais em tempo imaginário $t=-i \hbar \beta$ ao propagador deste campo escalar. $\mathrm{Na}$ realidade o que estava sendo feito era representar o operador densidade do sistema em equilíbrio a temperatura $T=\left(k_{B} \beta\right)^{-1}$.

Desenvolvendo a integral funcional resultante pelo método de fase estacionária, foi possível encontrar a parte imaginária da energia por unidade de volume da configuração metaestável e, consequentemente, computar sua taxa de decaimento para a configuração estável.

Apesar de a lagrangiana (3) não representar um campo escalar, ela possui um número infinito de graus de liberdade e, como tal, apresenta-se como boa candidata ao mesmo tipo de abordagem. A diferença é que devemos computar o operador densidade em equilíbrio para o sistema composto e posteriormente tomar o traço parcial com respeito às variáveis do reservatório para obter o operador densidade reduzido da partícula em equilíbrio a temperatura $T=\left(k_{B} \beta\right)^{-1}$ seguindo os mesmos passos que Feynman 16 em seu livro de mecânica estatística.

O operador densidade reduzido do sistema de interesse que resulta deste procedimento aplicado ao modelo descrito em (38) é 5,7

$$
\tilde{\rho}(x, y, \beta)=\tilde{\rho}_{0}(\beta) \int_{y}^{x} \mathcal{D} q(\tau) \exp -\frac{1}{\hbar} S_{e f f}[q(\tau)]
$$

onde

$$
\begin{aligned}
& S_{e f f}[q(\tau)]=\int_{0}^{\hbar \beta} d \tau\left\{\frac{1}{2} M \dot{q}^{2}+V(q)\right\}+ \\
& +\frac{1}{2} \int_{-\infty}^{+\infty} d \tau^{\prime} \int_{0}^{\hbar \beta} d \tau \alpha\left(\tau-\tau^{\prime}\right)\left\{q(\tau)-q\left(\tau^{\prime}\right)\right\}^{2}
\end{aligned}
$$

$$
\alpha\left(\tau-\tau^{\prime}\right)=\frac{\eta}{2 \pi} \frac{1}{\left(\tau-\tau^{\prime}\right)^{2}} .
$$

Esta integral funcional pode ser resolvida aproximadamente usando o método de fase estacionária e daí obtémse a parte imaginária da energia do estado fundamental (no caso de $T=0$ ) do sistema composto que pode ser associada à sua taxa de decaimento via tunelamento. Esta, por sua vez, pode ser interpretada como a probabilidade de tunelamento da partícula de interesse sob a influência do reservatório e é dada por

$$
\Gamma=A \exp -\frac{B}{\hbar}
$$

onde $B$ é a ação efetiva 24 computada na solução semiclássica do problema variacional $\delta S_{\text {eff }}[q(\tau)] / \delta q(\tau)=$ 0 e $A$ representa as flutuações quânticas em torno deste caminho em tempo imaginário [5,7].

O que é surpreendente neste resultado é que tanto $A$ quanto $B$ podem ser descritos apenas em função das constantes fenomenológicas que aparecem na equação (1) sem necessidade de se apelar para uma modelagem microscópica mais realista. Desta forma, a taxa de tunelamento do problema dissipativo depende dos parâmetros do potencial metestável ao qual a partícula está sujeita e ainda da constante de dissipação $\eta$, o que nos permite comparar diretamente as taxas $\Gamma_{0}$, do tunelamento sem dissipação, com a do tunelamento dissipativo, $\Gamma(\eta)$. No caso da dissipação linear com a velocidade (dissipação ohmica), pode-se mostrar que $\Gamma<\Gamma_{0}[5,7]$.

$O$ resultado acima mencionado pode ainda ser generalizado para o caso de temperaturas finitas e a conclusão permanece a mesma, a saber; a dissipação ohmica tende a inibir o proceso de tunelamento, ou seja, tende a tornar o sistema "mais clássico".

Alguns anos após a publicação dos primeiros resultados sobre o tunelamento dissipativo sua confirmação experimental foi obtida por John Clarke e colaboradores 17 investigando o comportamento da diferença de fase da função de onda supercondutora entre os terminais de uma junção Josephson sujeita a uma corrente externa.

\subsection{Tunelamento dissipativo: biestabilidade}

No exemplo acima, o potencial que atua na partícula browniana possui mínimos locais de energia que são metaestáveis, ou seja, há outros mínimos da energia potencial que têm energia menor que o mínimo correspondente à 
sua posição inicial. Entretanto, há casos em que os parâmetros que controlam a equação clássica de movimento nos fornecem um potencial externo biestável. É, por exemplo, o caso de um SQUID sujeito a um campo magnético externo cujo fluxo no interior do anel supercondutor é metade do quantum de fluxo $\phi_{0}$ 7]. Aqui o potencial $U(\phi)$ em (2) pode ser aproximado por $-a \phi^{2}+b \phi^{4}(a, b>0)$ onde há dois mínimos de energia degenerados.

Neste caso devemos nos lembrar que, na ausência de dissipação, os autoestados deste problema são pares ou ímpares na troca $\phi \leftrightarrow-\phi$ e podem ser obtidos através de combinações simétricas e antissimétricas de estados degenerados em energia localizados à direita $\left(\psi_{R}\right)$ ou à esquerda $\left(\psi_{L}\right)$ da barreira de potencial como

$$
\psi_{E}=\frac{1}{\sqrt{2}}\left(\psi_{R}+\psi_{L}\right) \quad \text { e } \quad \psi_{O}=\frac{1}{\sqrt{2}}\left(\psi_{R}-\psi_{L}\right) .
$$

Devido à taxa de tunelamento através da barreira de potencial ser finita, estas combinações (que seriam degeneradas na ausência de tunelamento) apresentam uma separação de energia exponencialmente pequena; $\hbar \Delta_{0}=$ $E_{O}-E_{E} \propto\left\langle\psi_{R}|\mathcal{H}| \psi_{L}\right\rangle=\left\langle\psi_{L}|\mathcal{H}| \psi_{R}\right\rangle$. Esta energia é muito menor que a separação de energia entre os estados fundamental e excitado de cada lado da berreira de potencial e, portanto, a baixas temperaturas, esperamos que apenas este subespaço bidimensional do sistema de interesse seja relevante no estudo da sua dinâmica quântica.

Considerando que o sistema de interesse esteja acoplado a um conjunto de osciladores harmônicos devemos recalcular e pequena separação de energia entre os dois estados acima mencionados usando a chamada renormalização adiabática [18], que consiste na inclusão de osciladores de alta frequência na construção dos novos autoestados (27) de forma autoconsistente. Os novos autoestados podem ser escritos como

$$
\Psi_{ \pm}\left(q,\left\{x_{j}\right\}\right)=\frac{1}{\sqrt{2}}\left[\Psi_{R}\left(q,\left\{x_{j}\right\}\right) \pm \Psi_{L}\left(q,\left\{x_{j}\right\}\right)\right],
$$

onde o conjunto $\left\{x_{j}\right\}$ representa as coordenas dos osciladores de alta frequência que podem ser eliminados em favor da obtenção de uma nova hamiltoniana efetiva para o universo. O método utilizado neste procedimento é novamente o da representação do operador densidade reduzido por integrais funcionais [18], anteriormente utilizado no cálculo da taxa de decaimento de um estado metaestável por tunelamento quântico.

A hamiltoniana resultante deste processo é

$$
\begin{aligned}
& \mathcal{H}=-\frac{1}{2} \hbar \Delta \sigma_{x}+\frac{1}{2} q_{0} \sigma_{z} \sum_{k} C_{k} q_{k} \\
& +\sum_{k} \frac{p_{k}^{2}}{2 m_{k}}+\sum_{k} \frac{1}{2} m_{k} \omega_{k}^{2} q_{k}^{2},
\end{aligned}
$$

conhecida como hamiltoniana spin-bóson, e que descreve basicamente um "spin" acoplado a um banho de osciladores. O spin a que nos referimos é apenas um sistema de dois níveis (sítios) que representam as posições biestáveis do potencial original. Aqui, $|\uparrow\rangle$ e $|\downarrow\rangle$ representam, respectivamente, os estados $\psi_{R}$ e $\psi_{L}$.

De posse desta hamiltoniana podemos estudar o movimento da partícula entre as duas configurações biestáveis em tempo real, o que nos permite analisar como a coerência quântica evolui neste processo dinâmico. Estabelecendo-se uma representação de trajetória seccionalmente constante para o movimento de saltos entre os dois sítios em questão podemos usar novamente a abordagem de Feynman e Vernon para estudar esta dinâmica dissipativa.

O resultado obtido no limite de dissipação fraca é o mesmo que se obteria tratando o problema de forma semifenomenológica através das equações de Bloch [7,18]. Definindo as componentes médias do "spin" como $S_{i}=$ $\hbar\left\langle\sigma_{i}\right\rangle / 2$, e $S_{x}^{(e q)}=\hbar \tanh (\hbar \beta \Delta) / 2$ como o valor médio da componente $S_{x}$ no equilíbrio termodinâmico, temos

$$
\begin{aligned}
& \frac{d S_{x}}{d t}=-\frac{S_{x}-S_{x}^{(e q)}}{T_{1}}, \\
& \frac{d S_{y}}{d t}=\Delta S_{z}-\frac{S_{y}}{T_{2}}, \\
& \frac{d S_{z}}{d t}=-\Delta S_{y},
\end{aligned}
$$

que são as mesmas equações usadas no problema da relaxação de um spin sujeito a um campo externo na direção $\hat{x}$. Neste caso particular em que se conhece a origem da dissipação, os tempos de relaxação longitudinal $\left(T_{1}\right)$ e transverso $\left(T_{2}\right)$ podem ser escritos em termos dos parâmetros do modelo como

$$
\frac{1}{T_{1}}=\frac{1}{T_{2}}=\frac{q_{0}^{2}}{2 \hbar} J(\Delta) \operatorname{coth} \frac{\beta \hbar \Delta}{2} .
$$

A dinâmica da partícula é fortemente dependente dos valores dos parâmetros do modelo. A evolução temporal de $S_{z}$, por exemplo, pode ser representada por uma oscilação amortecida, relaxação exponencial ou até mesmo pelo bloqueio da sua configuração original, dependendo da relação obedecida por $\Delta, \eta$ e $T$.

Convém notar que apesar de (29) representar uma hamiltoniana efetiva para a dinâmica dissipativa de uma partícula em um potencial biestável, ela pode ser usada para qualquer sistema de dois níveis não isolado do seu meio ambiente. Há ainda inúmeras conexões deste modelo com outros já bem conhecidos em física como, por exemplo, o utilizado no estudo do efeito Kondo [18.

\section{Computação quântica}

O desenvolvimento do estudo dos fenômenos quânticos dissipativos deu-se basicamente na primeira metade dos anos 1980. Em 1984, foi organizado um longo proprama sobre efeitos quânticos macroscópicos no então ITP (hoje KITP, Kavli Institute for Theoretical Physics) na Universidade da Califórnia em Santa Bárbara . Tratava-se 
de um programa de aproximadamente 9 meses que reuniu vários nomes envolvidos em temas que poderiam ser relacionados com a linha mestra da pesquisa a ser desenvolvida no evento. Fundamentos de mecânica quântica, ótica quântica e física de dispositivos supercondutores eram os assuntos mais populares. A propósito, havia ainda muitos debates sobre a aplicação da teoria descrita anteriormente sobre o tunelamento dissipativo no caso do tunelamento de estados de fluxo nos SDUIDs ou da fase da função de onda supercondutora em junções Josephson.

A abertura e o encerramento do programa foram marcados pela organização de duas oficinas de trabalho. A primeira foi basicamente um aquecimento para aqueles que já haviam chegado para o evento mais longo, mas a de encerramento foi mais grandiosa. Vários pesquisadores de renome foram convidados para contribuir para o evento e a última palestra foi reservada para ninguém menos que Richard Feynman.

Evidentemente um pesquisador de seu quilate é bemvindo em qualquer evento para falar sobre qualquer assunto e naquele, em particular, ele poderia apresentar algo perfeitamente inserido na temática do programa. Afinal, muito do que estávamos produzindo e estudando baseava-se tanto na sua formulação da mecânica quântica quanto no seu trabalho seminal com Frank Vernon sobre meios dissipativos lineares. Entretanto, não foi o que ocorreu, pelo menos à primeira vista; Feynman falou sobre computação quântica.

$\mathrm{O}$ assunto era para mim completamente novo e apesar de não ver conexão imediata com os temas que vínhamos debatendo ao longo daqueles 9 meses, resolvi usufruir daquela oportunidade única de ver Dick Feynman em ação, apresentando suas ideias sobre algo novo e absolutamente inusitado.

Apesar do meu desconhecimento sobre o assunto, já havia na época alguns poucos trabalhos que tinham sido publicados [19] na direção da construção de um computador quântico, mas, como vim a saber mais tarde, nem o prório Feynman sabia de sua existência quando começou a tratar do assunto. Novamente o velho fenômeno ocorria nesta area de pesquisa. Feynman havia desenvolvido todas as suas ideias sobre o tema simplesmente levantando algumas questões fundamentais que envolviam conjuntamente física e teoria da computação, como veremos adiante.

\subsection{Simulação de sistemas quânticos}

Feynman publicou apenas 2 trabalhos sobre computação quântica [20 21] e ambos tornaram-se referências na area. No primeiro 20], Feynman aborda a questão da simulação de sistemas físicos em geral.

A pergunta que Feynman procura responder é: Que tipo de computador devemos usar para simular o comportamento de um sistema físico? Como sabemos que em última instância a teoria que rege o comportamento de todos os sistemas físicos é a mecânica quântica, a questão se transforma em; que tipo de computador deve ser utilizado na simulação de sistemas quânticos?

Evidentemente podemos desenvolver poderosos algoritmos de solução de equações diferenciais para resolver aproximadamente a equação de Schrödinger em inúmeras situações de interesse, mas este não era exatamente o problema em que Feynman estava interessado. A sua pergunta buscava o tipo de processador universal, com componentes conectados localmente, que fosse capaz de imitar o comportamento de um sistema quântico cujos elementos interagiassem também localmente uns com os outros. Desta forma o seu processador imitaria a evolução no espaço - tempo de um determinado número de variáveis quânticas através de um número finito de operações lógicas nos seus elementos.

A análise desenvolvida por Feynman para solucionar este problema envolve questões extremamente complexas que vão desde a discretização do espaço - tempo até a reversibilidade das leis básicas da física. Há ainda a questão da simulação eficiente de um determinado processo que nos restringe a processadores com um número de elementos que não escale exponencialmente com o aumento do volume do espaço - tempo do sistema quântico de interesse.

Após uma longa e sofisticada argumentação ele conclui que a imitação eficiente de um sistema quântico só pode ser levada a cabo por um processador cujos elementos sejam também quânticos. Convém notar que processadores de computadores convencionais também possuem elementos quânticos, mas as operações realizadas obedecem a uma lógica puramente clássica. Ou seja, são efeitos quânticos que regem os processos dinâmicos que acarretam as respostas 0 ou 1 em um sistema binário. Detalhes sobre este ponto serão apresentados em seguida.

\subsection{Computadores quânticos}

O segundo trabalho que Feynman publicou nesta area 21 foi sobre o computador quântico propriamente dito. Da mesma forma que no caso anterior, um problema muito bem definido o inspirou nesta linha de pesquisa e este foi o da computação reversível [22 24] e da limitação de tamanho dos computadores face às leis fundamentais da física. Evidentemente não pretendo reproduzir neste texto toda a argumentação de Feynman sobre computadores quânticos, até porque sua notação diverge da comumente usada hoje em dia, mas vou procurar resumir de uma forma bem pedestre a essência dos problemas envolvidos nesta area.

Os computadores convencionais funcionam com base na lógica binária. Por exemplo, geração, armazenamento de mensagens ou operações matemáticas são executadas pela codificação dos elementos a serem usados por números de um sistema binário. Portanto, todos os símbolos que precisamos são sequências dos dígitos 0 e 1 . 
Para fazermos composições ou operações com essas sequências, precisamos gerá-las fisicamente. Desta forma, cada dígito da sequência deverá ser gerado por algum elemento físico que, quando excitado, responda com $0 \mathrm{ou}$ 1. Esses são os chamados bits de informação. Dentre as possibilidades de implementação dos bits podemos citar, por exemplo, um pequeno circuito elétrico que conduza corrente elétrica se sujeito a uma voltagem maior que um determinado valor, mas que não produza qualquer corrente se a voltagem for menor que esse valor de referência. Nesse caso, associamos o dígito 1 à presença de corrente e 0 à sua ausência. Se acoplarmos vários desses elementos, podemos gerar a sequência que quisermos. Todos os computadores que conhecemos, independentemente de sua complexidade, baseiam-se nestas idéias simples.

Evidentemente quanto maior o número de bits, maior será nossa capacidade computacional e este processo está baseado na miniaturização dos dispositivos usados nos computadores convencionais. Desnecessário dizer que isso se reflete no tamanho das máquinas disponíveis no mercado hoje em dia. Um simples laptop tem atualmente uma capacidade computacional muito maior que grandes máquinas da década de 1960. Este processo se deve a avanços relativos à manipulação da matéria em escalas de comprimento que já chegam a nanômetros.

Neste ponto uma das questões levantadas por Feynman ganha relevância. Cada operação em um único bit pode gerar uma variação de entropia de $k_{B} \log 2$ que, por sua vez, gera uma variação de calor $k_{B} T \log 2$ se o elemento encontra-se a temperatura $T$. Quando o número de elementos cresce nos deparamos com uma enorme produção de calor. Por exemplo, um processador composto por transistores pode dissipar algo da ordem de $10^{10} k_{B} T$ por operação lógica.

Por outro lado, nessa escala de comprimento, sabemos que os efeitos quânticos começam a se tornar importantes. O efeito quântico de interesse imediato está presente em sistemas que respondem com 0 ou 1 a uma determinada excitação, mas que, se convenientemente miniaturizados, apresentam uma sobreposição (ou superposição) dessas possíveis realizações. Mais ainda, estes seriam elementos ideais que não apresentariam qualquer variação de energia livre 22$]$.

Estes novos elementos são os chamados bits quânticos, ou simplesmente q-bits, e o seu estado físico é representado pela sobreposição dos 0 e 1 quânticos que são estados ortogonais de um sistema quântico de dois níveis. Uma forma bem conhecida de representá-los é através dos spinores $|\uparrow\rangle$ e $|\downarrow\rangle$ e o estado do q-bit pode ser escrito como

$$
|\psi\rangle=a|\uparrow\rangle+b|\downarrow\rangle .
$$

Uma consequência imediata deste princípio é a possibilidade da sobreposição de alternativas, ou seja, seqüências de "zeros" e "uns" que resultariam de bits convencionais podem agora coexistir entre várias realizações. Por exemplo, as seqüências 001000111..., 111000111..., 000001111..., etc. podem ocorrer simultaneamente. Quan- ticamente podemos escrever este estado como

$$
|\psi\rangle=\sum_{i, j, k, l \ldots} a_{i j k l \ldots}|i, j, k, l, \ldots\rangle,
$$

onde $i, j, k, l \ldots=\uparrow$ ou $\downarrow$. Este estado quântico, também conhecido como estado emaranhado, possibilitaria se fazer a chamada computação paralela com um único processador quântico.

Diante desta breve introdução poderíamos perguntar se não seria apropriado usar sistemas atômicos como q-bits. Apesar de a resposta ser positiva, acessar q-bits na escala atômica ou molecular não é uma tarefa simples. Por isso mesmo, inúmeros pesquisadores defendem o uso de dispositivos de dimensões extremamente reduzidas, mas que ainda possam ser acessados pela eletrônica dos processadores convencionais. Um dos candidatos que melhor se encaixa nessa descrição é o SQUID, o dispositivo supercondutor que apresentamos no exemplo do movimento browniano (2) no início deste trabalho .

Como vimos anteriormente, quando sujeito a um campo magnético externo de determinado valor, o SQUID apresenta duas configurações estáveis do fluxo magnético total que o atravessa. A temperaturas muito baixas e dissipação nula, há uma sobreposição destes estados biestáveis o que implica em que a corrente supercondutora no anel pode estar também em uma sobreposição de diferentes estados de movimento. Uma vez preparado em uma determinada configuração o sistema pode oscilar coerentemente entre suas duas configurações biestáveis. A este fenômeno dá-se o nome de coerência quântica macroscópica e os SQUIDs onde este efeito se manifesta são os chamados q-bits de fluxo.

Entretanto, há outro efeito extremamente importante que já estudamos na seção de tunelamento em um sistema biestável, mas que até o momento estivemos desprezando. Devido ao seu tamanho (ainda que extremamente pequeno), os SQUIDs acessíveis à eletrônica convencional sofrem forte influência do meio em que são construídos ou até mesmo do ruído intrínseco (flutuação) da corrente dissipativa que transportam juntamente com a corrente supercondutora. Essa influência aleatória e incontrolável pode ser mimetizada pela presença de termos de dissipação e flutuação presentes na equação (2) e o resultado da influência destes termos foi analisada anteriormente na seção do tunelamento dissipativo. A conclusão é que o meio ambiente destrói a coerência quântica necessária para a preservação da coexistência das diferentes configurações de corrente, e o SQUID pode tornar-se um bit convencional em um intervalo de tempo muito curto. Esse efeito é mais um exemplo de decoerência, um dos maiores empecilhos para a viabilização da computação quântica por q-bits supercondutores.

Apesar de ter apresentado um candidato muito particular para desempenhar o papel de um q-bit, convém enfatizar que esta não é a única possibilidade. Há inúmeros candidatos oriundos de diferentes áreas da física que 
podem apresentar vantagens e desvantagens em relação aos SQUIDs.

\section{Conclusões}

Neste trabalho procurei fazer um resumo do desenvolvimento recente nas áreas de dissipação e computação quântica mostrando como Feynman contribuiu para cada uma delas.

No caso da dissipação quântica, sua contribuição principal foi através de seu trabalho com Frank Vernon [9] onde a aplicação da representação de integrais funcionais para propagadores se mostrou extremamente útil na descrição da evolução temporal de operadores densidade reduzidos de subsistemas em tempo real. Posteriormente a utilização desta representação, tanto em tempo real como em tempo imaginário, foi usada com grande sucesso na resolução de problemas envolvendo o tunelamento quântico em sistemas dissipativos.

Já na computação quântica sua contribuição foi mais fundamental, apesar de bem menos quantitativa, e envolveu conjecturas (hoje em dia plenamente confirmadas) sobre simulação quântica e a própria proposta de se substituir os bits convencionais por q-bits.

Apesar de a criação ou o desenvolvimento destas áreas não poderem ser atribuídos exclusivamente a Feynman, não há dúvidas que suas contribuições foram fundamentais em ambas.

\section{Agradecimentos}

Agradeço ao Prof. Nelson Studart o convite para contribuir para esta edição especial da Revista Brasileira de Ensino de Física.

\section{Referências}

[1] R.P. Feynman and A.R. Hibbs, Quantum Mechanics and Path Integrals (McGraw-Hill, New York, 1965).

[2] R.P. Feynman, A.J.G. Hey and R.W. Allen, Feynman Lectures on Computation (Addison-Wesley, New York, 1996).

[3] B.D. Josephson, Physics Letters 1, 251 (1962).

[4] A.J. Leggett, Quantum Liquids: Bose Condensation And Cooper Pairing in Condensed-matter Systems (Oxford University Press, Oxford, 2006).

[5] A.O. Caldeira and A.J. Leggett, Annals of Physics 149, 374 (1983).

[6] A.O. Caldeira and A.J. Leggett, Physica A: Statistical Mechanics and its Applications 121, 587 (1983).

[7] A.O. Caldeira, An Introduction to Macroscopic Quantum Phenomena and Quantum Dissipation (Cambridge University Press, Cambridge, 2014).

[8] W.H. Louisell, Radiation and Noise in Quantum Electronics (McGraw-Hill, New York, 1964).

[9] R.P. Feynman and F.L. Vernon Jr., Annals of Physics 24, 118 (1963).
[10] H.P. Breuer and F. Petruccione, The Theory of Open Quantum Systems (Oxford University Press, Oxford, 2002).

[11] U. Weiss, Quantum Dissipative Systems (World Scientific, Singapore, 1999).

[12] A.O. Caldeira and A.J. Leggett, Phys. Rev. A 31, 1059 (1985).

[13] D. Forster, Hydrodynamic Fluctuations, Broken Symmetry, and Correlation Functions (Perseus Books, New York, 1990).

[14] S. Coleman, Phys. Rev. D 15, 2929 (1977).

[15] C.G. Callan and S. Coleman, Phys. Rev. D 16, 1762 (1977).

[16] R.P. Feynman, Statistical Mechanics: A Set Of Lectures Advanced Book Classics (Westview Press, Boulder, 1998).

[17] J. Clarke, A.N. Cleland, M.H. Devoret, D. Esteve and J.M. Martinis, Science 239, 992 (1988).

[18] A.J. Leggett, S. Chakravarty, A.T. Dorsey, M.P.A. Fisher, A. Garg and W. Zwerger, Rev. Mod. Phys. 59, 1 (1987).

[19] P. Benioff, Phys. Rev. Lett. 48, 1581 (1982).

[20] R.P. Feynman, Int. Journal of Theo. Phys 21, 467 (1982).

[21] R. P. Feynman, Optics News 11, 11 (1985).

[22] C. H. Bennett, IBM Journal of Research and Development 6, 525 (1979).

[23] C. H. Bennett, Int. Journal of Theo. Phys 21, 905 (1982).

[24] E. Fredkin and T. Toffoli, Int. Journal of Theo. Phys 21, 219 (1982). 